\title{
Correction: Effect of Collimator Angles on the Dosimetric Results of Volumetric Modulated Arc Therapy Planning for Patients with a Locally-Advanced Nasopharyngeal Carcinoma
}

\author{
[J. Korean Phys. Soc. 70, 539 (2017)] \\ DOI: $10.3938 / j k p s .70 .539$ \\ Yong Ho Kiм \\ Department of Radiation Oncology, Pusan National University Hospital, Busan 49241, Korea and \\ Department of Interdisciplinary Program in Biomedical Engineering, Pusan National University, Busan 49241, Korea \\ Dahl PArk, Ha Ryung PARK, Won Taek Kim, Dong Hyun Kim and Jin Suk BAE \\ Department of Radiation Oncology, Pusan National University Hospital, Busan 49241, Korea \\ Gye Rok JEON and Jung Hoon Ro \\ Department of Interdisciplinary Program in Biomedical Engineering, \\ Pusan National University, Busan 49241, Korea \\ Yongkan $\mathrm{KI}^{*}$ \\ Medical Research Institute, Pusan National University Hospital, Busan 49241, Korea and \\ Department of Radiation Oncology, Pusan National University Hospital, Busan 49241, Korea
}

DOI: $10.3938 /$ jkps. 75.748

The affiliation of Yongkan Ki should be corrected as follow:

Yongkan KI

Medical Research Institute, Pusan National University Hospital, Busan 49241, Korea and Department of Radiation Oncology, Pusan National University School of Medicine, Busan 49241, Korea

*E-mail: apex7171@hanmail.net; Fax: +82-51-248-5747 